\title{
CORRECTIONS TO "COMPLETE CONVERGENCE AND CONVERGENCE RATES FOR RANDOMLY INDEXED PARTIAL SUMS WITH AN APPLICATION TO SOME FIRST PASSAGE TIMES"
}

\author{
A. GUT (Uppsala)
}

In Section 5 of my paper [1] it is claimed that "by using obvious modifications" of previous proofs and the corresponding results in the classical case "one can obtain results", which are then given as Theorems 5.1 and 5.2. However, whereas the classical results corresponding to the results in the earlier sections are valid without restrictions on $\varepsilon$, this is no longer the case in Section 5 and the latter results therefore have to be modified slightly. The corrected results are presented below.

THEOREM 5.1. a) Let $r>2$. Suppose that $E X_{1}=0, E X_{1}^{2}=\sigma^{2}$ and $E\left|X_{1}\right|^{r}$. $\cdot\left(\log ^{+}\left|X_{1}\right|\right)^{-r / 2}<\infty$. If

$$
\sum_{n=1}^{\infty} n^{(r / 2)-2} P\left(\left|N_{n}-N n\right|>n \delta\right)<\infty \text { for all } \delta>0
$$

where $N$ is a positive random variable, such that $P(N \geqq A)=1$ for some $A>0$, then

$$
\sum_{n=1}^{\infty} n^{(r / 2)-2} P\left(\left|S_{N_{n}}\right|>\varepsilon \sqrt{N_{n} \log ^{+} N_{n}}\right)<\infty, \quad \varepsilon>\sigma \sqrt{r-2}
$$

b) Let $r=2$. Suppose that $E X_{1}=0$ and $E X_{1}^{2}<\infty$. If (5.1) holds with $N$ as above, then (5.2) holds for all $\varepsilon>0$.

c) Let $r \geqq 2$. Suppose that $E X_{1}^{2}=\sigma^{2}<\infty$ and $E X_{1}=0$. If (5.1) holds with $P(N \leqq B)=1$ for some $B>0$, then

$$
\sum_{n=1}^{\infty} n^{(r / 2)-2} P\left(\left|S_{N_{n}}\right|>\varepsilon \sqrt{n \log n}\right)<\infty, \quad \varepsilon>\sigma \sqrt{(r-2) B}
$$

THEOREM 5.2. a) Suppose that $E X_{1}=0, E X_{1}^{2}=\sigma^{2}$ and that

$$
E X_{1}^{2} \frac{\log ^{+}\left|X_{1}\right|}{\log ^{+} \log ^{+}\left|X_{1}\right|}<\infty
$$

If

$$
\sum_{n=1}^{\infty} \frac{1}{n} P\left(\left|N_{n}-N n\right|>n \delta\right)<\infty \text { for all } \delta>0
$$

where $N$ is a positive random variable, such that $P(N \geqq A)=1$ for some $A>0$, then

$$
\sum_{n=1}^{\infty} \frac{1}{n} P\left(\left|S_{N_{n}}\right|>\varepsilon \sqrt{N_{n} \log ^{+} \log ^{+} N_{n}}\right)<\infty, \quad \varepsilon>2 \sigma
$$


However, if (5.4) holds with $P(A \leqq N \leqq B)=1$ for $0<A \leqq B<\infty$, then $E X_{1}^{2}=$ $=\sigma^{2}<\infty$ and $E X_{1}=0$ imply that (5.5) holds for $\varepsilon>\sigma \sqrt{2 \frac{B}{A}}$.

b) Suppose that $E X_{1}^{2}=\sigma^{2}<\infty$ and $E X_{1}=0$. If $(5.4)$ holds with $P(N \leqq B)=1$ for some $B=0$, then

$$
\sum_{n=3}^{\infty} \frac{1}{n} P\left(\left|S_{N_{n}}\right|>\varepsilon \sqrt{n \log \log n}\right)<\infty, \quad \varepsilon>\sigma \sqrt{2 B} .
$$

REMARRK. A minor change should also be made in Theorem 2.1, namely that the assumption should be "If, for some $\delta>0$, (2.1) holds (with $\varepsilon$ replaced by $\delta$ )" and the conclusion should be that "(2.2) holds for all $\varepsilon>0$ ". As a consequence some $\varepsilon$ have to be replaced with $\delta$ in an obvious way.

Finally, as a consequence of the above facts, the formulas in part $\mathbf{C}$ on page 231 (which furthermore should have been numbered as (5.7)-(5.9)) are valid for all $\varepsilon>\sigma \sqrt{2 \mu^{-3}}, \sigma \sqrt{2}$ and $\sigma \sqrt{2 \mu^{-1}}$, respectively, and formulas (5.8) and (5.9) in D should have been called (5.10) and (5.11).

\section{Reference}

[1] A. Gut, Complete convergence and convergence rates for randomly indexed partial sums with an application to some first passage times, Acta Math. Hung., 42 (1983), 225-232.

\section{(Received Febrtary 15, 1984)}

UPPSALA UNIVERSITY

DEPARTMENT OF MATHEMATICS

THUNBERGSVÄGEN 3

S-752 38 UPPSALA

SWEDEN 